\title{
Consumo de ÁlCoOl entre eStudantes de uMA ESCOLA PÚblica da CidAde de Cajazeiras, PB*
}

\section{Gilberto Santos Cerqueira'; Cicera Tavares de Lucena ${ }^{2}$; Ana Teresa de Morais Gomes ${ }^{2}$; Ana Paula Fragoso de Freitas ${ }^{3}$; Nayrton Flávio Moura Rocha ${ }^{1}$; Saulo Rios Mariz ${ }^{4}$}

O objetivo deste trabalho foi identificar o uso de bebidas alcoólicas pelos estudantes da maior escola pública da cidade de Cajazeiras, PB. Realizou-se estudo descritivo transversal, com abordagem quantitativa, e a amostra ficou composta por 300 alunos de uma escola pública da cidade. Verificou-se que 71\% já tinham usado álcool, 66,4\% fizeram experimentação da droga entre 13-17 anos, 69,4\% usaram por diversão, 59,5\% já se embriagaram ao consumir álcool, 21,3\% experimentam em bares/boates e 39,4\% beberam com maior frequência em bares/danceterias/boates. Constatou-se que há alto consumo de álcool entre adolescentes, sendo necessária a implementação de ações educativas, visando a diminuição do consumo.

Descritores: Alcoolismo; Adolescentes; Drogas Ilícitas.

\section{Alcohol use among students of a public school in Cajazeiras, Pernambuco*}

The objective of this study was to identify the use of alcohol among students of the largest public school in Cajazeiras, Pernambuco. This cross-sectional descriptive study was performed with a sample of 300 students. It was found that $71 \%$ had drunk alcohol before; $66.4 \%$ had their first drink when they were between $13-17$ years old; $69.4 \%$ drank for fun; 59.5\% have already gotten drink from consuming alcohol; $21.3 \%$ had their first drink at bars/night clubs; 39.4\% usually drank at bars/dance clubs/night clubs. It was found that alcohol use is high among adolescents, thus there is a need to implement educational actions, aiming at reducing alcohol use.

Descriptors: Alcoholism; Adolescent; Street Drugs.

\footnotetext{
*Pesquisa realizada com auxílio financeiro da Faculdade de Enfermagem Santa Maria

1-Farmacêuticos, Doutorandos em Farmacologia, Departamento de Fisiologia e Farmacologia, Faculdade de Medicina, Universidade Federal do Ceará, Fortaleza, CE, Brasil. E-mail: giufarmacia@hotmail.com.

2-Alunas do Curso de Bacharel em Enfermagem, Voluntárias de Iniciação Científica, Faculdade de Enfermagem Santa Maria, Cajazeiras, PB, Brasil.

3-Enfermeira, Mestranda, Programa de Pós-graduação em Ciências Médicas da Universidade Federal do Ceará. E-mail: paulinhaff4@hotmail.com.

4-Farmacêutico-Bioquímico, Doutor em Farmacologia, Professor Adjunto II, Unidade Acadêmica de Medicina, Universidade Federal de Campina Grande, PB, Brasil. E-mail: sjmariz22@hotmail.com.

Endereço para Correspondência:

Gilberto Santos Cerqueira

Rua Delmiro de Farias, 1517, Bairro Rodolfo Téofilo

CEP 60430-170. Fortaleza, CE, Brasil

E-mail: giufarmacia@hotmail.com
} 


\section{CONSUMO DE ALCOHOL EN ESTUdiANTES DE UNA ESCUELA PÚBLICA DE LA Ciudad de Cajazeiras, PB*}

El objetivo de este trabajo fue identificar el uso de bebidas alcohólicas por parte de estudiantes de la mayor escuela pública de la ciudad de Cajazeiras-PB. Se realizó un estudio descriptivo transversal con abordaje cuantitativo, sobre muestra de 300 alumnos de una escuela pública de la ciudad. Se verificó que $71 \%$ ya había consumido alcohol; $66,4 \%$ experimentó con drogas entre los 13-17 años; $21,3 \%$ tuvieron experiencias en bares/discotecas; 39,4\% bebieron con mayor frecuencia en bares/discobares/discotecas. Se constató que hay alto consumo de alcohol entre adolescentes, haciéndose necesaria la implementación de acciones educativas, apuntando a la disminución del consumo.

Descriptores: Alcohol; Adolescentes; Drogas Ilícitas 


\section{Introdução}

O álcool, por ser droga lícita, é amplamente tolerado pela sociedade, e o jovem tem, muitas vezes, sua primeira experiência de consumo dentro da própria família, através de hábitos culturais ou sob a forma de diversão.

O consumo de álcool, tabaco e outras drogas regulamentadas está aumentando e contribuindo, de maneira evidente, para a carga de doenças em todo o mundo. No caso do álcool, embora o nível de consumo nos últimos vinte anos tenha diminuído nos países desenvolvidos, está aumentando nos países em desenvolvimento ${ }^{(1)}$.

Estudos mostram que o envolvimento com "drogas ilícitas e lícitas" ocorre principalmente dentro da população de adolescentes e adultos jovens ${ }^{(2)}$. No Brasil, onde 35 milhões de pessoas têm menos de 30 anos, os problemas relacionados ao consumo de substâncias psicoativas podem ser preocupantes ${ }^{(3-4)}$. Algumas pesquisas, apresentadas a seguir, vêm sendo desenvolvidas com populações específicas, dentre as quais, os adolescentes

Pesquisas científicas demonstram que o uso do álcool começa na infância. Assim, a família tem grande responsabilidade no que diz respeito ao contato inicial da criança com essa droga. Nas famílias onde não se vivencia situações de violência doméstica, em que existe diálogo sobre os problemas do cotidiano, onde há interesse dos pais pelos filhos é comprovado que existe menor probabilidade do uso abusivo de álcool ${ }^{(5)}$.

Uma das maiores preocupações dos pais, atualmente, é se seu filho irá desenvolver episódios violentos e/ou usar substâncias psicoativas, visto que os adolescentes são considerados como grupo especialmente vulnerável à experimentação de álcool, tabaco e outras drogas, e isso tem levado muitos pesquisadores a afirmar que tal fase da vida merece ser acompanhada com especial atenção( ${ }^{(6)}$.

A adolescência é etapa na qual, frequentemente, ocorre a experimentação de drogas, sejam elas lícitas ou ilícitas. Embora, na maioria das vezes, esse uso seja apenas experimental, é possível notar padrões de uso que irão perdurar até a vida adulta e que podem ser indicativos da necessidade de estabelecer medidas preventivas nessa fase do desenvolvimento humano(7). Estudiosos relatam que há informações consistentes sobre elementos que influenciam o início ou mantêm o uso de substâncias psicoativas, por parte do adolescente, podendo-se citar o fato de ele ter amigos que usam drogas, gerando pressão de grupo em direção ao uso. Elementos relacionados à estrutura de vida do adolescente desempenham papel fundamental na progressão de um padrão de uso experimental frequente ou compulsivo, ou seja, na gênese da dependência de drogas. Como exemplos, podem ser citadas situações de brigas constantes no lar, separação dos pais e influência de $\operatorname{amigos}^{(8)}$.

O uso nocivo de bebidas alcoólicas por adolescentes tem despertado o interesse de profissionais de diversas áreas, com isso, surgem múltiplas estratégias de intervenção na tentativa de reduzir os problemas associados a esse comportamento $^{(6)}$.
No sentido de buscar melhores maneiras de intervir nessa realidade, bem como o grande número de acidentes de trânsito, envolvendo adolescentes alcoolizados, na cidade de Cajazeiras (PB), surgiu o interesse de se desenvolver pesquisas sobre esse assunto, objetivando analisar o uso de bebidas alcoólicas pelos estudantes da maior escola pública da cidade de Cajazeiras, PB. De modo mais específico, a intenção foi identificar as principais bebidas utilizadas pelos estudantes, bem como as características desse uso, de modo a otimizar a implantação de ações preventivas que sejam eficazes na redução do consumo de álcool entre os estudantes.

\section{Metodologia}

Trata-se de estudo transversal, com abordagem quantitativa. O cenário da investigação deu-se em uma escola pública estadual, situada na cidade de Cajazeiras, PB. Essa cidade é um dos principais municípios da Paraíba. Situado na extremidade ocidental do Estado, Cajazeiras é atualmente a cidade que mais cresce na Paraíba, segundo o Instituto Brasileiro de Geografia e Estatística ${ }^{(2)}$.

A população do estudo constituiu-se de adolescentes estudantes no maior colégio da cidade. A Escola possui cerca de 2.368 alunos matriculados, sendo que o presente estudo teve, como amostra, 300 alunos selecionados de forma aleatória, e, dessa maneira, foi obtida, inicialmente, como amostra ideal para o desenvolvimento deste estudo $^{(9)}$.

Para a coleta de dados entre os estudantes selecionados para a pesquisa, aplicou-se um questionário baseado no modelo do Centro Brasileiro de Informação sobre Drogas Psicotrópicas (Cebrid) ${ }^{(10-11)}$, através de autopreenchimento e de modo sigiloso. A equipe básica para coleta de dados foi composta por dois estudantes e dois pesquisadores, previamente treinados para a aplicação do questionário. Os dados foram coletados entre março e junho de 2007.

A análise dos dados foi do tipo descritivo, a fim de determinar o perfil sociodemográfico da amostra estudada, comportamento e consequências do consumo de álcool. $\mathrm{O}$ teste $\mathrm{t}$ de Student e o teste do qui-quadrado $\left(\mathrm{c}^{2}\right)$ foram aplicados para verificar a associação entre as variáveis estudadas, no nível de significância de 5\%. Foi utilizado para organização do banco de dados o programa de computador Excel, versão 2003, e como instrumento de análise estatística o aplicativo Graph Pad Prisma, versão 5.0.

Este estudo foi aprovado pelo Comitê de Ética em Pesquisa do Hospital Universitário Lauro Wanderley da Universidade Federal da Paraíba (Protocolo n ${ }^{\circ}$ 67/2008). Participaram do estudo adolescentes cujos responsáveis legais assinaram o termo de consentimento. Além disso, o diretor da escola assinou um documento permitindo a realização do estudo. Essa pesquisa não possui nenhum conflito de interesses e segue os preceitos da Resolução 196/96 do Conselho Nacional de Saúde, norma que regulamenta a pesquisa envolvendo seres humanos ${ }^{(12)}$. 


\section{Resultados}

A pesquisa evidenciou que a maioria dos estudantes estava matriculada no período diurno $(78,7 \%)$, eram do gênero feminino $(54,2 \%)$. Em relação à faixa etária, o maior índice era de jovens entre 16 e 18 anos com 46,3\%, sendo a segunda faixa entre 13 e 15 anos com $32,3 \%$.

Verificou-se que $70 \%$ dos participantes da pesquisa consumiramálcool,não existindo diferença estatisticamente significante no consumo de álcool em relação ao gênero $\left(X^{2}=0,48, \mathrm{p}=0,49\right)$ (Tabela 1$)$.

Tabela 1 - Distribuição da frequência de consumo de álcool, segundo gênero, entre estudantes. Cajazeiras, 2007

\begin{tabular}{lcccccccc}
\hline \multicolumn{8}{c}{ Consumo de álcool } \\
& \multicolumn{2}{c}{ Sim } & & \multicolumn{2}{c}{ Não } & & \\
\cline { 2 - 3 } & $\mathbf{n}$ & $\%$ & & $\mathbf{n}$ & $\%$ & $\mathbf{X}^{2}$ & p-valor \\
\hline Masculino & 100 & 72,99 & & 37 & 27,01 & 0,48 & 0,49 \\
Feminino & 113 & 69,33 & & 50 & 30,67 & & \\
\hline
\end{tabular}

Valores significativos $\mathrm{p}<0,05$ pelo teste do qui-quadrado

Quanto ao uso de álcooll nos últimos seis meses foi relatado por $71 \%$ dos indivíduos da amostra total. Desses, verificou-se que 59,5 44 dos entrevistados já consumiram álcool até a embriaguez, não existindo diferença estatisticamente significante $(p<0,2672)$ entre esses e aquelesestudantes que consumiram álcool e nunca se embriagaram (Tabela 2).

Entre os estudantes investigados, 69,5 \pm 45 (56\%) relataram que os pais possuíam conhecimento que seus filhos consumiamálcool. Dessa forma, observa-se diferença estatisticamente significante $(\mathrm{p}<0,0306)$ em relação aos pais que conheciam o consumo de álcool pelos filhos, quando comparado aos pais que não sabiam que os filhos consumiam álcool. No que concerne ao conhecimento dos pais em relação ao consumo de álcool, verificou-se que $69,5 \pm 45(56 \%)$ dos pais possuíam conhecimento que seus filhos consumiam essa droga, dessa forma, observase diferença estatisticamente significante $(p<0,0306) \mathrm{em}$ relação aos pais que conheciam e aqueles que os pais não sabiam que os filhos consumiam álcool.

Tabela 2 - Distribuição da estatística descritivas em relação ao consumo de álcool e a presença da embriaguez entre estudantes. Cajazeiras, 2007

\begin{tabular}{lccc}
\hline Consumo de álcool com & Média \pm erro padrão & Mínimo & Máximo \\
\hline Embriaguez & $59,50 \pm 4,5$ & 55 & 64 \\
Sem embriaguez & $52,00 \pm 2,0$ & 50 & 64 \\
\hline
\end{tabular}

Valores representam a médiaterro padrão da média.

A bebida mais consumida foi a cerveja, com 39,4\%, seguida dos vinhos $(36,9 \%)$, 9,2\% uísque e, em quarto lugar, as bebidas destiladas com $7,4 \%$ da preferência. O licor aparece em último lugar, com 1,5\%. O tipo de bebida utilizada não foi especificado em $3 \%$ dos casos, de acordo com a Tabela 3 .
Tabela 3 - Distribuição da frequência das principias bebidas consumidas entre estudantes de Cajazeiras, 2007

\begin{tabular}{|c|c|c|}
\hline Bebida & $\mathrm{n}$ & $\%$ \\
\hline Cerveja & 118 & 39,4 \\
\hline Uísque & 28 & 9,2 \\
\hline Destilados & 22 & 7,4 \\
\hline Vodka & 15 & 4,9 \\
\hline Vinhos & 12 & 4 \\
\hline Sidra & 6 & 2 \\
\hline Não consumiu & 4 & 1,5 \\
\hline Diversos & 9 & 3 \\
\hline Não informou & 86 & 28,6 \\
\hline
\end{tabular}

No que se refere à utilização de algum outro tipo de droga, durante o consumo de álcool, a grande maioria $(74,3 \%)$ afirmou já ter adotado tal prática, enquanto o restante $(25,7 \%)$ relatou nunca ter feito uso de qualquer tipo de droga concomitante ao álcool. As principais drogas utilizadas foram lança-perfume, maconha e solventes.

Quanto ao contexto do primeiro uso, 21,3\% dos adolescentes relataram que foi em bares e boates. Em seguida, está a opção "na casa de amigos" (15,7\%), e com os familiares $(15,3 \%)$, em festas realizadas em domicílios (Tabela 4).

Tabela 4 - Distribuição da frequência em relação ao local do consumo de álcool entre os estudantes de Cajazeiras, 2007

\begin{tabular}{lccc}
\hline & Local & $\mathbf{n}$ & $\%$ \\
\hline Bares/boates & 64 & 21,3 \\
Casa de amigos & 47 & 15,7 \\
Lar & 46 & 15,3 \\
Não informaram & 77 & 25,7 \\
\hline
\end{tabular}

A Tabela 5 ilustra os fatores que levam os adolescentes a usar bebidas alcoólicas, onde foram obtidos os seguintes resultados: 69,4\% bebem por diversão, 18,8\% afirmaram que bebem por influência dos companheiros, $6,1 \%$ por motivos não citados, $4,3 \%$ por gostar dos efeitos e 1,6\% por problemas com a família.

Tabela 5 - Distribuição da frequencia dos estudantes em relação aos fatores que influenciaram o consumo de álcool na cidade de Cajazeiras, 2007

\begin{tabular}{lcc}
\hline \multicolumn{1}{c}{ Fatores que influenciaram } & $\mathbf{n}$ & $\%$ \\
\hline Diversão & 208 & 69,4 \\
Companheiros & 56 & 18,8 \\
Fissura & 13 & 4,3 \\
Problemas familiares & 5 & 1,6 \\
Outros & 18 & 6,1 \\
\hline
\end{tabular}

\section{Discussão}

Como se pode verificar, há elevado relato de experimentação de álcool entre os adolescentes da escola pública da cidade de Cajazeiras, avaliados neste estudo. Esta pesquisa corrobora a de Souza e colaboradores (2005) 
que encontraram prevalência de $71,3 \%$ do consumo de álcool entre adolescentes da rede estadual de ensino de Cuiabá, Mato Grosso ${ }^{(12)}$. Esses dados estão coerentes com aqueles encontrados nos quatro levantamentos realizados no Brasil, nos anos 1987, 1989 e 1997, os quais relatam experimentação de álcool acima de $65 \%$ entre jovens escolares brasileiros ${ }^{(13)}$. Outro estudo ${ }^{(14)}$ encontrou, em população de adolescentes de 12 a 17 anos, prevalência de uso de álcool, no último ano, de 32,7\%.

Resultados semelhantes aos de Cajazeiras foram encontrados em adolescentes da cidade de Ribeirão Preto, quando se constatou índice de uso de álcool em torno de $89,2 \%{ }^{(15)}$.

O II levantamento domiciliar sobre uso de Drogas Psicotrópicas no Brasil, estudo envolvendo as 108 maiores cidades do país, realizado pelo Centro Brasileiro de Informações sobre Drogas Psicotrópicas (Cebrid), com indivíduos entre 12 e 25 anos, constatou que 12,3\% da população brasileira é dependente do álcool. Entre esses, existe maior prevalência de homens com 19,5\%, do que de mulheres $(6,9 \%)^{(11,16)}$.

O primeiro levantamento domiciliar sobre o uso de drogas, no Brasil, mostra prevalência de dependentes de álcool, em adolescentes de 12 a 17 anos, de 5,2\%, e que esse número tende a aumentar em pessoas dessa faixa etária com transtornos mentais e de conduta ${ }^{(11,17)}$.

A embriaguez é problema não somente de alcoolistas como também um risco para usuários que estão apenas experimentando, ou seja, aqueles que estão no início da escala evolutiva de utilização, pois, nesse estado, há grande alteração da fisiologia do corpo humano, como depressão do sistema nervoso central e inibição da gliconeogênese hepática, responsável em grande parte pela hipoglicemia alcoólica. É muito comum, na cidade de Cajazeiras, PB, encontrarem-se adolescentes embriagados na rua durante os finais de semana. Em estudos no interior paulista, 24\% dos estudantes relataram já haver bebido até se embriagar em algum momento da vida, e $19,5 \%$ reportaram embriaguez nos últimos 12 meses $^{(18)}$

Muitos adolescentes consomem álcool sem que seus pais saibam ou, muitas vezes, o problema está em casa, quando um parente é o estimulante do consumo. Quanto à família, estudos mostram que o uso nocivo de álcool pelos pais e a falta de controle e supervisão sobre o consumo dos filhos associam-se ao aumento do risco de uso nocivo e dependência na idade adulta ${ }^{(19-20)}$.

$\mathrm{O}$ componente ambiental, relacionado à família e amigos, pode determinar, de modo geral, a ocorrência de alcoolistas $^{(21)}$, seja por uso indiscriminado, enfraquecimento nas relações familiares, rejeição dos pais aos filhos, entre outros. Dessa forma, pode-se inferir que o beber entre os familiares estimula, direta ou indiretamente, o consumo de substâncias entre os jovens e, consequentemente, aumenta o risco de problemas relacionados ao uso de álcool tais como embriaguez e alcoolismo.

O papel dos pais e do ambiente familiar é marcante no desenvolvimento do adolescente e, consequentemente, na sua relação com álcool e outras drogas. Falta de suporte parental, uso de drogas pelos próprios pais, atitudes permissivas dos pais perante o uso de drogas, incapacidade de controle dos filhos pelos pais, indisciplina e uso de drogas pelos irmãos são todos fatores predisponentes à maior iniciação ou continuação do uso de drogas por parte dos adolescentes ${ }^{(8)}$.

De acordo com o levantamento sobre os padrões de consumo de álcool na população brasileira, a cerveja e/ou chope são as bebidas mais consumidas pelos brasileiros quando se comparam bebidas pelo número de doses consumidas, anualmente. Em estudo realizado no interior de São Paulo, na cidade de Paulínia, a cerveja foi a bebida mais consumida pelos estudantes (40\%), seguida de vinhos (36,9\%) e, em terceiro lugar, as bebidas tipo ice, com 10,2\% da preferência. Resultados semelhantes foram encontrados neste estudo. Nos dias atuais, as propagandas de bebida exercem forte influência no estímulo do consumo. Na cidade de São Bernardo do Campo, SP, pesquisadores identificaram que $79 \%$ dos adolescentes consumiram bebidas alcoólicas após exposição prévia às propagandas com essas bebidas.

O uso e o abuso de álcool e outras drogas têm sido uma das principais causas desencadeadoras de situações de vulnerabilidade na adolescência e juventude, a exemplo dos acidentes, suicídios, violência, gravidez não planejada e a transmissão de doenças por via sexual e endovenosa, nos casos das drogas injetáveis. Não fosse o consumo de drogas problema suficientemente grave, há, ainda, a problemática do uso de drogas nas escolas, representando, no Brasil e em outros países, séria ameaça à estabilidade $\operatorname{social}^{(22)}$.

Dentre as variáveis que influenciaram o consumo de álcool, evidenciou-se que momentos de diversão, como festas e comemorações, são os principais fatores que influenciam o consumo. Nessas situações, os adolescentes geralmente estão acompanhados de companheiros consumidores, relatam desejo compulsivo pelo álcool e experimentam problemas familiares. Entre alunos consumidores de álcool do Estado de São Paulo, observouse que $28,8 \%$ dos estudantes possuem algum familiar que bebeu a ponto de causar problema, no último ano, e, ainda, que $7,6 \%$ dos consumidores indicou o pai como sendo esse familiar, $3,1 \%$ o irmão e $19,1 \%$ outro familiar ${ }^{(23-24)}$.

\section{Considerações Finais}

O alcoolismo é doença e um dos mais graves problemas de saúde pública encontrado no mundo, uma vez que é observado hoje em grande escala nas várias populações. Para a farmacodependência ao álcool não há distinção de raça, cor, religião ou de nível social, já que acomete todos. Tal enfermidade pode gerar ainda outros diversos agravos à saúde do ser humano.

Diante dos estudos feitos, pode-se observar que o uso de álcool em adolescentes escolares é, na maioria dos casos, experimental, mas deve ser acompanhado de perto e desestimulado por diversos fatores.

O consumo precoce do uso de álcool é fato importante e que deve ser levado em consideração na escolha dos momentos mais adequados ao início de abordagens 
preventivas. Os amigos e companheiros desempenham papel relevante na introdução do jovem ao consumo de drogas.

De acordo com os resultados obtidos, identificouse alta prevalência de consumo experimental de álcool entre escolares, sendo necessária a implantação de ações educativas nessa população, visando a diminuição do consumo de álcool entre adolescentes da cidade de Cajazeiras.

\section{Agradecimentos}

Os autores agradecem a Débora Pamplona, da Casa de Cultura Inglesa da UFC, pela correção do abstract, e Patrícia Mastroianni e Sebastian Orellana, da Unesp Araraquara e da Universidade do Chile, respectivamente, pela correção do resumen.

\section{Referencias}

1. Lopes GT, Villar LMA. A formação do enfermeiro e o fenômeno das drogas no estado do Rio de Janeiro Brasil: atitudes e crenças. Rev. Latino-Am. Enfermagem. 2005;13(n.esp): 872-9.

2. Instituto Brasileiro de Geografia e Estatística - IBGE. Projeção da população do Brasil por sexo e idade para o período de 1980-2050: revisão. Rio de Janeiro; 2004.

3. Kandel DB, Yamaguchi K. From beer to crack: developmental patterns of drug involvement. Am J Public Health. 1993;83(6):851-5.

4. Silva LVER, Malbergier A, Stempliuk, VA, Andrade AG de. Fatores associados ao consumo de álcool e drogas entre estudantes universitários. Rev Saúde Pública. 2006;40(2):280-8.

5. Alavarse, G.MAC, Barros, MD. Álcool e adolescência: o perfil de consumidores de um município do norte do Paraná. Esc Anna Nery. 2006;3(10):408-16.

6. De Micheli D, Fisberg M, Formigoni ML. Estudo da efetividade da internação breve para o uso de álcool e outra drogas em adolescentes atendidos num serviço de assistência à saúde. Rev Assoc Méd Bras. 2004;50(3):30513.

7. Tavares BF, Béria JU, Lima MS. Prevalência do uso de drogas e desempenho escolar entre adolescentes. Rev Saúde Pública. 2001;35(2):40-6.

8. Pechansky F, Szobot CM, Volettos SCI. Uso de álcool entre adolescentes: Conceitos, caracterização epidemiológicas e fatores etiopatogênicos. Rev Brás Psquiatr. 2004;26 supl 1:14-7.

9. Oliveira EFTI de, Grácio MCC. Análise a respeito do tamanho de amostras aleatórias simples: uma aplicação na área de Ciência da Informação. DataGramaZero: Rev Ciênc Informação. 2005. jun;6(3).[acesso 12 out 2009]. Disponível em: http://dgz.org.br/ago05/Art 01.htm.

10. Galduroz JCF, Noto AR, Fonseca AM, Carlini EA. $\mathrm{V}$ levantamento nacional sobre o consumo de drogas psicotrópicas entre estudantes do ensino fundamental e médio da rede pública de ensino nas 27 capitais brasileiras: 2004. São Paulo: Centro Brasileiro de informações sobre Drogas psicotrópicas, UNIFESP; 2005.

11. Galduroz JCF, Caetano R. Epidemiology of alcohol use in Brazil. Rev Bras Psiquiatr. 2004;26(Supl 1):3-6.

12. Ministério da Saúde (BR). Conselho Nacional de Saúde. Comitê de Ética em Pesquisa em Seres Humanos. Resolução no 196, de 10 de outubro de 1996. Diretrizes e Normas Regulamentadoras Envolvendo Seres Humanos. Brasília (DF); 1997.

13. Figlie N, Fontes A, Moraes E, Payá R. Filhos de dependentes químicos com fatores de risco biopsicossociais: necessitam de um olhar especial? Rev Psiquiatr Clín. 2004;31(2):53-62.

14. Galduróz JCF, Nodo AR, Carlini EA. IV levantamento sobre o uso de drogas entre estudantes de $1^{\circ}$ e $2^{\circ}$ graus em 10 capitais brasileiras, 1997. São Paulo: Centro Brasileiro de Informações sobre Drogas Psicotrópicas, Departamento de Psicobiologia da Universidade Federal de São Paulo; 1997. p. 130.

15. Wu LT, Schlenger WE, Galvin DM. The relationship between employment and substance use among students aged 12 to 17. J Adolesc Health. 2003;32:5-15.

16. Muza GM, Bettiol H, Muccillo G, Barbieri MA. Consumo de substâncias psicoativas por adolescentes escolares de Ribeirão Preto, SP (Brasil). I - Prevalência do consumo por sexo, idade e tipo de substância. Rev Saúde Pública. 1997;31(1):21-9.

17. Kessler F, Von Diemen L, Seganfredo AC, Brandão I, Saibro P, Scheidt B, et al. Psicodinâmica do adolescente envolvido com drogas. Rev Psiquiatr Rio Gd Sul 2003;25(Supl 1):33-41.

18. Vieira DL, Ribeiro M, Romano M, Laranjeira RR. Álcool e adolescentes: estudo para implementar políticas municipais. Rev Saúde Pública. 2007;41(3):396-403.

19. Grunbaum JA, Kann L, Kinchen S, Ross J, Hawkins J, Lowry R, et al. Youth risk behavior surveillance-United States, 2003. MMWR Surveill Summ. 2004;53(2):1-96.

20. Kuntsche E, Rehm J, Gmel G. Characteristics of binge drinkers in Europe. Soc Sci Med. 2004;59(1):113-27.

21. Pillon SC. O uso de álcool e a educação formal dos enfermeiros. [Tese]. São Paulo: Universidade Federal de São Paulo. Escola Paulista de Medicina. Programa de PósGraduação em Psiquiatria e Psicologia Médica; 2003.

22 . Vendrame, A, Pinsky, I, Faria, Silva, R. Apreciação de propagandas de cerveja por adolescentes: relações com a exposição prévia às mesmas e o consumo de álcool. Cad. Saúde Pública. 2009 25(2): 359-365.

23. Almeida, MM, Oliveira, MA, PINHO, PH. O tratamento de adolescentes usuários de álcool e outras drogas: uma questão a ser debatida com os adolescentes? Rev Psiquiatr Clín., São Paulo 2008. [acesso 29 set 2008]. Disponível em: http://www.scielo.br/scielo.php?script=sci_arttext\&pi $\mathrm{d}=\mathrm{S} 010160832008000700016 \& \operatorname{lng}=\mathrm{pt} \& \mathrm{nrm}=$ iso.

24. Martins, RA, Cruz, LAN, Teixeira, OS et al., Padrão de 
consumo de álcool entre estudantes do ensino médio de uma cidade do interior do estado de São Paulo. SMAD, Rev. Eletr Saúde Mental Álcool Drog. (Ed. port.). [online]. fev. 2008, 4(1). [acesso 29 set 2008]. Disponível em URLhttp://pepsic. bvs-psi.org.br/scielo.php?script=sci_arttext\&pid=S1806-

$69762008000100005 \& \operatorname{lng}=$ pt\&nrm $=$ iso

\section{Como citar este artigo:}

Cerqueira GS, Lucenta CT, Gomes ATM, Freitas APF, Rocha NFM, Mariz SR. Consumo de álcool entre estudantes de uma escola pública da cidade de Cajazeiras, pb. SMAD, Rev. Eletrônica Saúde Mental Álcool Drog. (Ed. port.). jan-abr 2011 [acesso: ;7(1):18-24. Disponível em:

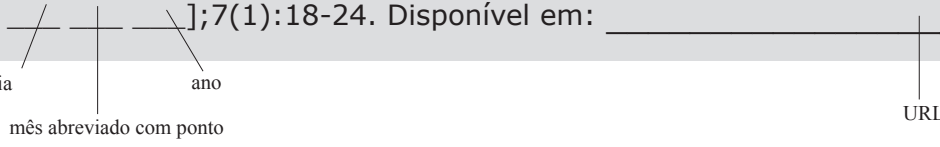

